\title{
LA FORMACIÓN DE COMPETENCIAS DIRECTIVAS MEDIANTE LA TRANSMISIÓN DE EXPERIENCIAS DE DIRECTORES RETIRADOS
}

Carlos La Bandera Trejo*

\section{Resumen}

La globalización obliga a incrementar la competitividad de las empresas, que resulta afectada cuando éstas aceleran el tiempo de retiro de sus directivos llevándose sus experiencias consigo. Al mismo tiempo, la ciencia médica busca mantener sanos a los adultos mayores a edades más avanzadas. Se presenta una paradoja entre ambas tendencias. Para solucionarla, incrementar la productividad y competitividad y evitar la pérdida de capital intelectual en la empresa, se propone un modelo de formación de competencias directivas aprovechando las experiencias de los directores retirados que, además, los revalora en beneficio de la sociedad. En este trabajo se presentará un análisis del contexto, del problema y del enfoque propuesto.

\section{Abstract}

Globalisation forces the increase of companies' competitiveness, which is affected when these accelerate the time of retirement of their managers taking their experience with themselves. At the same time, medical science looks after elders' health in late stages of life. A paradox between both tendencies appears. In order to solve it, increase the productivity and competitiveness and avoid the loss of intellectual capital in the company, a model of formation of directive competitions is set out taking advantage of the retired directors' experience, which, in addition revalue, them in benefit of the society. In this work, we will present an analysis of the context, of the problem and the proposed approach.

PALABRAS CLAVE: Competencias, transmisión de experiencias, ejecutivos de alto nivel, globalización.

* Doctorante en Educación por la Universidad La Salle Ciudad de México, Director del Centro de Desarrollo Ejecutivo de Ernst \& Young México, Estudios de postgrado en Minnesota Training \& Development Research Center, treinta y seis años de experiencia en el campo de la Educación de Adultos, fue presidente de Corporación Cultural y Publicataria LAR, S. carlos.la-bandera@mx.ey.com 


\section{Introducción}

La globalización ha incrementado la competencia en todos los órdenes y obliga a las empresas a optimizar al máximo todos sus recursos. El ritmo vertiginoso de la sociedad actual tiende a desechar todo lo que no le reporta una utilidad inmediata. Día con día, abandonan el mercado laboral personas en edad productiva y experimentadas, y cuando tienen la suerte de una jubilación y pensión, ingresan al sistema de seguridad social (elevando su carga económica). Así, de manera contradictoria con el eficientismo actualmente imperante, la sociedad y las empresas derrochan lo más valioso por irrecuperable: el activo humano que representan los trabajadores jubilados, particularmente ex directivos o personal altamente capacitado.

La hipótesis general de la investigación afirma que al desaprovechar el conocimiento surgido de la experiencia de los ex directivos, las empresas dejan de reducir costos, pierden competitividad y reducen su utilidad económica y social. Por tanto, se requiere el diseño, aplicación y evaluación de un modelo de enseñanza aprendizaje para enriquecer y acelerar la formación de competencias directivas mediante las experiencias de exdirectivos retirados, con la finalidad de aumentar la productividad, la competitividad y la utilidad social de las empresas.

El objetivo de esta ponencia es mostrar, entre los participantes al 1er. Congreso Nacional de Ciencias Humanas, la conveniencia de encontrar, en el marco de la globalización, nuevos paradigmas en el mundo empresarial en cuanto a la formación y actualización de las competencias de sus ejecutivos, aprovechando nuevos enfoques, los cuales muy probablemente, se deberán utilizar en un futuro más cercano de lo que pudiera pensarse.

La globalización de las empresas

El concepto de globalización propicia el estudio de "la naturaleza de las crisis globales". Es un nuevo término de fines del siglo XX que enuncia un cambio mundial en las relaciones totales: económicas, sociales y hasta políticas, de las regiones del mundo, países y compañías, tal como mencionan Naisbitt, J. y Aburdene, P. (1990, p.4). En este fenómeno mundial, se señala a la tecnología, especialmente de la información, como aspecto económico-social que penetra en el mundo industrializado del siglo $X X$ y afecta a las empresas profundamente, destacando la competitividad ante la cual, las empresas se obligan a incrementar su productividad (Bain, D. 1982). Se busca el mejoramiento de la productividad como instrumento para afrontarla (Drucker, P. 1993). Ese incremento de productividad está estrechamente relacionado con la toma de decisiones como competencia directiva para utilizar de la mejor manera posible los recursos 
escasos con que se cuenta (Gordon, T. 1980). Son los directivos, quienes tienen la responsabilidad de conducir a la empresa por un camino de productividad. Entonces, el capital humano toma cada vez mayor valor como factor productivo de la empresa, y se revalora su actividad como un capital propio de la misma empresa (Andrews, D. y Jonson, K. 2003).

Valor del capital humano

Hoy en día, puede decirse que la economía del conocimiento es resultado de la revolución científico-tecnológica [nota: sí se trata de un concepto compuesto] mundial, la cual revaloriza el conocimiento del ser humano y su potencialidad como el factor activo más importante en la producción. Ya no la información sino el conocimiento que utiliza y produce información para tomar decisiones. Esta nueva característica que definirá necesariamente nuestro siglo XXI denominada economía del conocimiento, será factótum del desarrollo global. Se vive la nueva era de la economía del conocimiento (Lladós, J. 2005). En general, el valor del capital humano se acrecienta cada vez más en las instituciones y en las sociedades gracias al valor del conocimiento como capital intelectual de la empresa. Las empresas hacen gran énfasis en contratar y mantener a las personas más preparadas y talentosas, ya que son e-llas quienes tienen la posibilidad de llevar a la empresa a destacar de su competencia (Gundling E. 2003).

No parece ser la labor operativa diaria del llamado capital humano en general la que consigue incrementos de productividad, sino las decisiones de los ejecutivos las que indican cómo y en dónde sí y en dónde no deben aplicarse los recursos para obtener los resultados esperados (Crosby, P. 1990). Y más aún, es en la esfera más alta de la empresa, donde se define la misión, visión, valores y estrategias de la compañía, el lugar al que corresponde la responsabilidad de la competitividad. Son entonces los directivos de empresas los que reafirman su valor ante la compañía por el conocimiento que obtienen de sus continuas experiencias y de su aplicación a través de la interpretación que realizan de sus vivencias apoyados en sus conocimientos previos (Díaz Barriga, F. y Hernández Rojas, G. 1998). Esto les genera un caudal de experiencias de las que las empresas se apropian a través del proceso de toma de decisiones.

Desaprovechamiento de los exdirectivos

Las empresas propician que los directores de altos niveles ejecutivos se retiren entre los sesenta y sesenta y cinco años en México. Inclusive, es posible que el retiro se produzca años antes. De hecho en los países desarrollados los mismos directivos particularmente desean retirarse desde años antes, alrededor de los 55 
a 58 años aunque lo logran en promedio a los 63 , para dedicarse a actividades bien diferentes a su función de directivos.

En este sentido es de destacarse la siguiente paradoja: mientras tanto, la ciencia médica avanza para apoyar la salud de los exdirectivos, ya que al mismo tiempo que dejan de "servir a la sociedad", se observa una fuerza encontrada que tiende a incrementar la vida de los adultos mayores en las mejores condiciones posibles de salud mental y física, impulsándolos a vivir más tiempo, como se muestra en el estudio realizado por Lozano y Frenk (1999). Esto parece una contradicción, pues lleva a cuestionar de inmediato el por qué no se ha buscado con intensidad al mismo tiempo, la forma de aprovechar al adulto mayor dentro de la sociedad productiva.

Exdirectivos retirados en México

Considerando a las empresas por tamaño en cuanto a número de empleados, para enero de 2006, las empresas en la zona metropolitana de la Ciudad de México con más de cien empleados eran más de 5 mil. $Y$ de éstas, las empresas con más de 500 personas empleadas consideradas como AAA por Mercamétrica (2006), eran 1,631 como se muestra en la tabla 1. A continuación se presenta la clasificación según el Sistema de Clasificación Industrial de América del Norte (SCIAN) (Tabla 1). 
TABLA 1: Número de empresas grandes en México

\begin{tabular}{|c|c|c|}
\hline Giro & $\begin{array}{l}\text { Número de empresas } \\
\text { con más de } 500 \\
\text { empleados }\end{array}$ & $\begin{array}{l}\text { Porcentaje } \\
\text { del total de } \\
\text { empresas }\end{array}$ \\
\hline Industrias & 824 & 50.5 \\
\hline Alimentarias & 306 & 18.8 \\
\hline Automotrices & 78 & 4.7 \\
\hline Enseres domésticos & 11 & 0.7 \\
\hline Materiales para construcción & 28 & 1.2 \\
\hline Del papel & 38 & 2.3 \\
\hline Del vidrio & 15 & 0.9 \\
\hline Eléctricas y electrónicas & 38 & 2.3 \\
\hline Extractivas & 12 & 0.7 \\
\hline Metalmetálicas & 52 & 3.1 \\
\hline Quimicas & 71 & 4.3 \\
\hline Sidenúrgicas & 11 & 0.7 \\
\hline Textiles & 66 & 4.0 \\
\hline Otras industrias & 98 & 6.0 \\
\hline Bancos y seguros (1) & 45 & 2.7 \\
\hline Cadenas de tiendas & 63 & 3.8 \\
\hline Empresas comerciales & 86 & 5.2 \\
\hline Empresas de servicios & 389 & 23.9 \\
\hline Constructoras & 83 & 5.0 \\
\hline Grupos & 141 & 8.7 \\
\hline TOTAL (2) & 1,631 & 100.0 \\
\hline
\end{tabular}

Tabla 1: Número de empresas grandes en la República Mexicana, con base en el número de empleados. Se consideran más de 500 personas para indicar que una empresa es AAA o grande.

(1): 86 bancos y seguros con más de cien empleados.

(2): 5.812 empresas con más de cien empleados.

Si de esta tabla se consideran 1631empresas grandes con alrededor de diez puestos directivos de alto nivel y una rotación del $10 \%$ anual, se tendrían en diez años del orden de 16 mil personas que pueden ser calificados como directivos retirados con salud, sujetos de esta investigación.

Desarrollo de recursos humanos de alto nivel 
Los especialistas, conforme avanzan en la escalera de responsabilidades escalando puestos, se les presenta la necesidad de contar con las características del ejecutivo (Covey, F. 1990). Requieren de una formación no sólo técnica, sino administrativa, inclusive diplomática, de relaciones en el mundo de los negocios pero principalmente la toma de decisiones con énfasis en el trato con inversionistas, dueños, accionistas, presidentes y directores de empresas clientes.

La experiencia generadora de conocimiento

Considérese la siguiente definición de experiencia tomada de Aristóteles (1475/1997): "La actividad que desarrolla conocimiento debido a la observación de la realidad y su comparación con las bases de conocimiento anterior con que cuenta el sujeto". Pero no todas las experiencias son interesantes para compartirlas. En el mundo empresarial, aquéllas experiencias que llevan a la adecuada toma de decisiones son las que valen la pena compartir, porque son experiencias irreemplazables que llevan a aplicar el conocimiento que generan en próximas situaciones similares, que se repiten en la labor directiva de los ejecutivos diariamente. Al conjunto de estas experiencias se les denomina: "experiencia ejecutiva". Las demás experiencias, las que no se aplican a la toma de decisiones dentro de la empresa, o no son de interés, o son experiencias de la aplicación de conocimientos exclusivamente técnicos, que se pueden encontrar en los libros y que por lo tanto, son objeto de estudio de otras ciencias. Se busca entonces, compartir las experiencias ejecutivas que implican un nuevo conocimiento generado por las experiencias (Heidegger, M. 1970), las que a su vez, significan una pérdida irreparable cuando el sujeto que obtuvo dichas experiencias se vaya a la tumba, pues habría que esperar a que otro ejecutivo tenga una experiencia similar para que produzca un conocimiento similar al que se haya perdido con la muerte del primer ejecutivo que no compartió su conocimiento sobre esa determinada experiencia.

Construcción de conocimiento tácito y explícito

Considerando los escritos de Nonaka y Takeuchi (1995) sobre la experiencia tácita y la experiencia tácita que aún no es encasillada (embodied) en un concepto, y su relación con la experiencia explícita que es susceptible de conformar una base de datos. Las vivencias de los individuos, y en este caso de los directivos y ejecutivos de empresas, generan conceptos que pasan a la memoria de corto plazo y de ahí a la memoria de largo plazo en la medida en que tienen prioridad a través de un proceso de justificación. El resultado es la creación de conocimiento tácito en el sujeto, sobre las vivencias y éstas relacionadas con los conceptos ya existentes en la memoria. Tal conocimiento se debe a la experiencia y se denomina conocimiento tácito. Éste, puede pasar a convertirse en conocimiento explícito, en 
la medida en que se relaciona con el medio ambiente y se utiliza para generar una reacción ante un estímulo externo. El resultado de este proceso es la creación de conocimiento explícito, el cual puede ser utilizado para nuevas acciones del sujeto, lo que implica como resultado nuevas vivencias que a su vez y a través del mismo proceso, dan origen a nuevas experiencias.

Estrategias para transferir el conocimiento basado en la experiencia

El conocimiento tácito se puede convertir en conocimiento explícito, tal como lo menciona Takeuchi a través de diversas estrategias de sucesión de la administración, lo que se denomina gestión del conocimiento, como lo indica Rodwell W. (2004) en su artículo sobre la captura de las lecciones de la experiencia en doce estrategias de las cuales se consideran tres:

1. Entrevistas y cuestionarios de incidentes críticos: se utiliza desde los años 50, cuando se presentan incidentes críticos en la empresa se documentan a través de cuestionarios o entrevistas a los que participaron en el incidente para conocer cómo sucedió y cuál fue su solución.

2. Programas de Mentoring (asesoría): un veterano que aconseja a una persona con menor experiencia, y que generalmente no debe ser su jefe para evitar intereses y egoísmos que pueden presentarse entre subalternos. (Ésta es una estrategia seleccionada para el caso).

3. Storytelling (anécdota): es una descripción de una situación que sucedió. Esta estrategia es la más usual para transferir la sabiduría de la empresa a otras personas.

Modelo propuesto: lineamientos generales

Una vez identificadas las causas por las cuales los exdirectivos no se aprovechan, se procede a diseñar un modelo de orientación cualitativa que permita el aprendizaje a los ejecutivos de las experiencias de los exdirectivos retirados y que contribuya a la explicación de cómo puede aprovecharse esa experiencia. El modelo tiene su cimentación en las teorías aplicables que en este caso se refiere a las investigaciones del constructivismo y del proceso para explicitar el conocimiento tácito que es propio de las experiencias. Este modelo aporta una fundamentación teórica a partir de recuperar la experiencia de los exdirectivos retirados, señalando las políticas para formación de ejecutivos y las acciones para otorgar viabilidad al modelo y la facilidad de su implementación práctica y posterior evaluación. La construcción del modelo se va dando paulatinamente como puede apreciarse, iniciando con un marco de referencia.

Características del modelo 
- Aprovechar a los exdirectivos en la empresa en que se retiraron:

Los exdirectivos son las personas con mayor conocimiento de la empresa y sus experiencias son resultado de la operación sui géneris de cada una de ellas en lo particular (Matthews, M. 1998).

Se reconoce que la probabilidad de aprovechamiento de los exdirectivos es más viable dentro de la propia empresa a la que ofrecieron sus servicios porque:

1. Conocen su contexto y la dinámica del negocio.

2. Cuentan con respeto de ejecutivos por haber sido directores de éstos.

3. Han asimilado las experiencias que pueden ser más aplicables a esa empresa considerando su misión, visión y valores.

4. Tienen la confianza para aportar sus ideas y experiencias debido a

su pasado y el nivel en el que se desempañaron.

La formación de ejecutivos fuera de la empresa implica siempre un riesgo de que no se aprenda conforme a los requerimientos del aquí y el ahora y que desvíe su atención en otros mecanismos que no sean aplicables a la realidad de su compañía. El modelo trata de aprovechar

las experiencias de los directivos retirados o jubilados de las empresas en un plano más cotidiano y regular, lo cual conlleva, al menos, cuatro beneficios:

1. Una actividad que puede mejorar substancialmente la toma de decisiones de ejecutivos más jóvenes y con menor experiencia.

2. Aprovechar el compartir los conocimientos de experiencias ejecutivas redunda en una mejor toma de decisiones en la empresa para acelerar su desarrollo, evita errores ya cometidos anteriormente e incrementa su productividad.

3. Impulsar la educación continua aprovechando con mayor ímpetu a los directivos retirados, para apoyar la formación y actualización de jóvenes que pretenden ser ejecutivos. Es decir, apoyar a los ejecuti- 
vos y a los que pueden llegar a serlo.

4. Facilitar en los adultos mayores exdirectivos retirados, un sentido

por el cual seguir desarrollándose, desde el momento en que se

convierten en un pilar para apoyar la formación de ejecutivos jóvenes,

situación que les provocaría un mayor cuidado de su salud física y

mental.

A manera de conclusiones y discusión: problemática del modelo a desarrollar

Uno de los problemas fundamentales a investigar es si los exdirectivos retirados, parecen no estar muy dispuestos a compartir sus experiencias debido a la fuerza de la costumbre social. Además, probablemente los ejecutivos que se encuentran ahora en funciones y que adolecen de dichas experiencias directivas, tampoco están muy dispuestos a otorgar credibilidad a los exdirectivos. Es, pues, necesario investigar si los directivos, cuando se retiran, tienen disposición real de transmitir sus experiencias como un conocimiento que han construido a través de sus vivencias durante muchos años de actividad y, al mismo tiempo, si los jóvenes en proceso de formación como ejecutivos muestran interés en aprender de las experiencias de otras personas con mayor edad afrontando el choque generacional. Asimismo, una vez que se obtiene la disposición de ambos sujetos es importante diseñar los mecanismos dirigidos a lograr una transmisión de experiencias de los exdirectivos retirados que provoquen un nuevo conocimiento aplicable por parte de los nuevos directivos a nuevas situaciones y no a las mismas.

Asimismo, hay una característica que vale la pena señalar a favor de la relación entre exdirectivos y ejecutivos: Como lo especifica (Ryan, D.1985) sobre la motivación aplicando la pirámide de Maslow, la re-lación se enriquece debido a la necesidad de reconocimiento por parte de ambos. Este beneficio, que pueden obtener los exdirectivos en su relación con ejecutivos, a través de los esfuerzos que se realicen para la formación de nuevos directivos, puede actuar como motivador de nuevas acciones entre los retirados exdirectivos que estarían más dispuestos a transmitir sus experiencias para apoyar indirectamente a la empresa que sirvieron. Lo mismo ocurre con los ejecutivos en formación, que presentan una admiración especial por los exdirectivos retirados de su empresa que una vez fueron sus líderes (Mc Cormark M. 1989) y que ahora pueden encontrar en ellos la sabiduría requerida para su formación y actualización.

\section{Fuentes de Consulta}


ANDREWS, D. y Jonson, K. (2003). Revolutionizing IT. The art of using information technology effectively. New York, USA: Barnes \& Noble

ARISTÓTELES, (1997), Ética Nicomaquea y Política, Madrid, España: Editorial Po-rrúa.

BAIN, D. (1982). Productividad, la solución a los problemas de la empresa. Ciudad de México: Mc Graw-Hill de México.

COVEY, F. (1990). Los siete hábitos de la gente altamente efectiva. Ciudad de México: Editorial Paidós Mexicana.

CROSBY, P. (1990). Liderazgo, el arte de convertirse en un ejecutivo. Ciudad de México: Mc Graw Hill/ Interamericana.

DÍAZ Barriga, F. y Hernández Rojas, G. (1998). Estrategias docentes para un aprendizaje significativo. Una interpretación constructivista. Ciudad de México, Mc Graw-Hill Interamericana.

DRUCKER, P. (1993). Gerencia para el futuro, el decenio de los 90 y más allá. Bogotá, Colombia: Grupo Editorial Norma.

GORDON, T. (1980) L.E.T. Líderes eficaz y técnicamente preparados. Método para liberar el potencial productivo de las personas. Ciudad de México: Editorial Diana.

GUNDLING, E. (2003). Working GlobeSmart, 12 people skills for doing business across borders. New York, USA: Barnes \& Noble.

HEIDEGGER, Martin, (1970), Hegel's Concept of Experience, New York, Harper \& Row, Publishers.

LLADÓS, J. (2005), La creación de empresas en la economía del conocimiento, en $X$ Jornada de Economía de Caixa Manresa (2004).

LOZANO R. y Frenk J. (1999), El Envejecimiento; sus Desafíos y Esperanzas, Ciudad de México, México: Siglo XXI Editores.

MC CORMARK M. (1989). Los secretos del éxito. Ciudad de México: Laser Press Mexicana.

MATTHEWS, M. (1998), What Lies Beyond Knowledege Managemet: Wisdom Creation and Versatility, Journal of Knowledge Management, (Vol. 1, March 1998, 207-214). 
Mercamétrica Ediciones, S.A. (2005) Industridata, Base de datos empresariales, Ciudad de México, México.

NAISBITT, J. y Aburdene, P. (1990). Mega tendencias 2000, diez nuevos rumbos para los años 90 . Ciudad de México, Editorial Norma..

NONAKA, I. and Takeuchi, H. (1995) The Knowledge Creation Company, New York, U.S.A., Oxford University Press.

ROTHWELL, W. (2004). Capturing the Lessons of Experience: Knowledge Transfer en Workforce Planning, Feb. 2004, Pennsylvania State University. EUA.

RYAN, D. (1985). Intrinsic Motivation and Self-Determination in human behavior. New York, USA; Three Falmer Press. 